\title{
Study on Intelligent Logistics Management Information System Based on IOT and Cloud Computation in Big Data Era
}

\author{
Yajun Guo* and Jinli Qu
}

Department of Computer Science, Tangshan Normal University, Tangshan Hebei 063000, China

\begin{abstract}
In this paper, we analyzed the influence of IOT and cloud computation on the development of the logistics industry, built the intelligent logistics system on the basis of IOT and cloud computation, analyzed the three-layer architecture of the system and at the end, predicted the great change to be caused by IOT and cloud computation for the logistics industry in the big data era.
\end{abstract}

Keywords: Big data, cloud computing, integration and share, internet of things, key geological area, service-oriented architecture.

\section{INTRODUCTION}

The data take place 'big explosion' under the promotion of development of Internet of Things, cloud computing and mobile Internet. Its scale rises exponentially and it enters the big data era using $\mathrm{ZB}$ as the basic unit $(1 \mathrm{ZB}=1024 \mathrm{~EB}=1024$ $X 1024 \mathrm{~PB}=1024$ X 1024 X 1024 TB). The hot focus of research is to dig 'big data' to mine some 'rich deposits' those hide its inner. It brings a series of challenges and impunities from data acquiring to transportation, storage, manipulation, share and analysis of data. The geological informationization level will improve much after integrated IOT and Cloud Computing together. This paper does a lot of study on domestic and foreign research conditions about big data, Internet of Things and cloud computing and then expands the research on these technologies in the geological survey field combining with the requirements of geological survey informationization. [1]

The framework of full process informationization is raised in this paper that from wild data collection to store, organization, and management and field application based on those technologies such as IOT and cloud computing and so on.

The whole framework and physical deployment of Geological Internet of Things (GIOT) is designed with the enough understanding of application status in geological survey field and needs of GIOT. Thinking about the application needs of national geological materials to propose to use RFID, sensor and robots to implement ordered intelligent management for geological material. This paper put forward a scientific solution to manage geological equipments based on IOT. This solution is applied to the first IOT project in Beijing "The system construction of geological disaster monitoring about highway high slope". Using some IOT devices such as udometer, tension meter and camera to collect data in the field, and then transfer data through the network. After have received the data, those can be showed in two or three dimension, filtered and early warning analysis. Finally the paper forecast the application trend in the geological survey field. [2]

Cloud computing is used as technology method, massive geological data storage management and analysis application as mote power, 'information find deposit' in prospecting breakthrough strategic action of MLR as holds for hands, the software and hardware environment of the key laboratory of geological information technology of MLR as cushion to raise the construction methods of geological cloud computing(GCC) platform as shown in Figs. (1) and (3). Then it designed the whole view and architecture of GCC. The geological cloud storage solution uses that one of visualization methods to realize the resource management of software and hardware. [3] The geological cloud computing platform (GCCP) is constructed based on Service-Oriented Architecture. The platform integrates and shares a great number of distributed web services with geological information 'One Map' concept and contributes the management methods for services ring and show tactics of data fusion. The services are issued by various servers that content of those comes from geological map, geological disasters, mining rights check and so on. The geological information one stop application (GIOSA) is programmed by rich Internet application technology and users can access the resources in the cloud through the network only without think about the data come from where or IOT.

Using the needs of mining rights edit and review of key geological exploration and business requirements of formline staff and experts to design the rules of reasonable analysis of mining rights set considering geological background.

The development and review aided system for mining rights set (DRASMRS) is developed by 'One Map' concept and it integrates geology, mineral, gravity, natural heavy minerals, and magnetic, remote sensing data into system smoothly. DRASMRS focus on the geological characteristics 


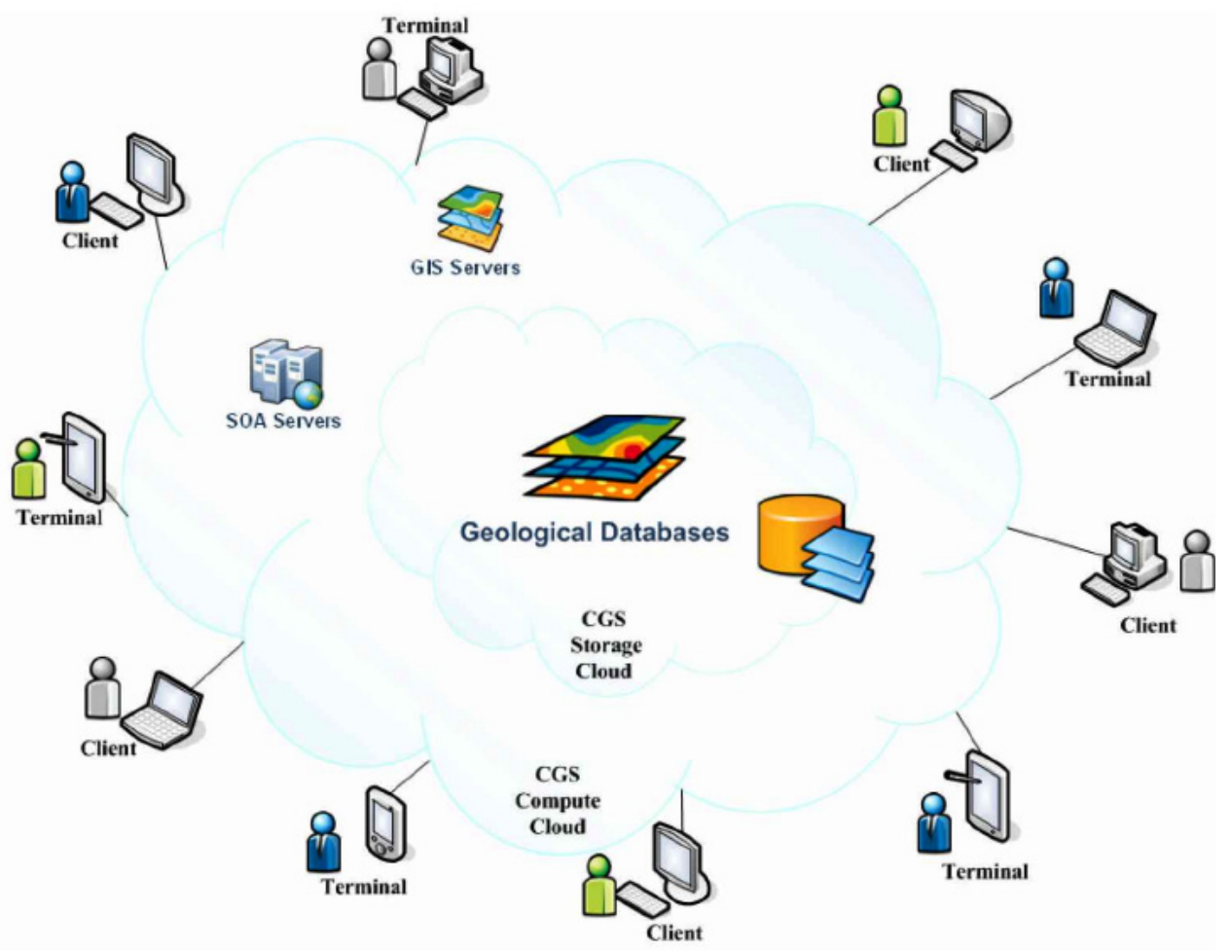

Fig. (1). Cloud computing platform.

and ore-controlling factors of deposits in the mining rights set area. The system completed the edit and review works of the second batch of 31 mining rights set solutions in the country. The results show the system can raise work efficiency and accuracy and scientific of mining rights set dramatically. The data check tool of mining rights check is designed and developed based on rule base. The tool is used in the nationwide and the data quality of about 150 thousand mining rights is guaranteed effectively [4].

\section{INTELLIGENT INFORMATION MANAGEMENT ARCHITECHTURE}

The Internet of Things (IOT) is the network which enables information exchange and communication among its terminals located at any objects connected by the Internet. For the first time, IOT combines the collection, transmission, process and application of the information. With the "Intelligence" as its main character, IOT has become one of the key driving forces for the economic growth, as shown in Fig. (2).

With the rapid development of global economy and science and technology, logistic industry, as one of important service industries in national economy, booms rapidly in the world and gradually, becomes an important fundamental industry in national economy. And its prosperity has become one of standards to measure the development of national economy. Logistics industry takes an important place in national economy. Through improving the managerial level and efficiency of logistics and reducing the logistics cost, logistics bring about a remarkable economic benefit to enterprises and the society. Meanwhile, it improves the operational efficiency of national economy and our international competitiveness.
The premise basis of TPL (Third-Part Logistics) intelligent lies in the development of IOT (Internet of Things) technology, global industrial chain division and cooperation and modern logistics industry development. The emergence of IOT technology makes TPL intelligent impossible. On one hand, the pursuit of celerity, high-efficiency and safety of global industrial chain division and cooperation and the Three Path more specialization and socialization of logistics demand are sure to promote intelligent of TPL. Therefore, through reduction of operating costs and the an intense competitiveness also exists in the enhancement of benefit efficiency, establishment of enterprise brand, in logistics system, TPL continues to search the resource integration and optimization leading to TPL possesses a strong internal driving force in pursuing of logistics intelligent. Just because of these factors, the research of TPL intelligent based on IOT technology has a reasonable feasibility and a realistic necessity, and its significance of study stands to reason.

Based on the thought of system engineering, the business process and the system structure of TPL will be introduced, and then an analysis of logistics intelligent mechanism and its features will be given. It should be noticed that to realize the logistics system intelligent, IOT technology must be taken into consideration. This paper, based on IOT technology and TPL study, makes a deep research on TPL system's business process and structure, analyzes TPL intelligent mechanism, and introduces the logistics intelligent mechanism on the basis of Agent. Meanwhile, the paper introduces the operating principle of expert system and intelligent decision support system. It proposes that IOT technology of information exchange must be relied on for achieving the ideal Agent logistics intelligent mechanism. 


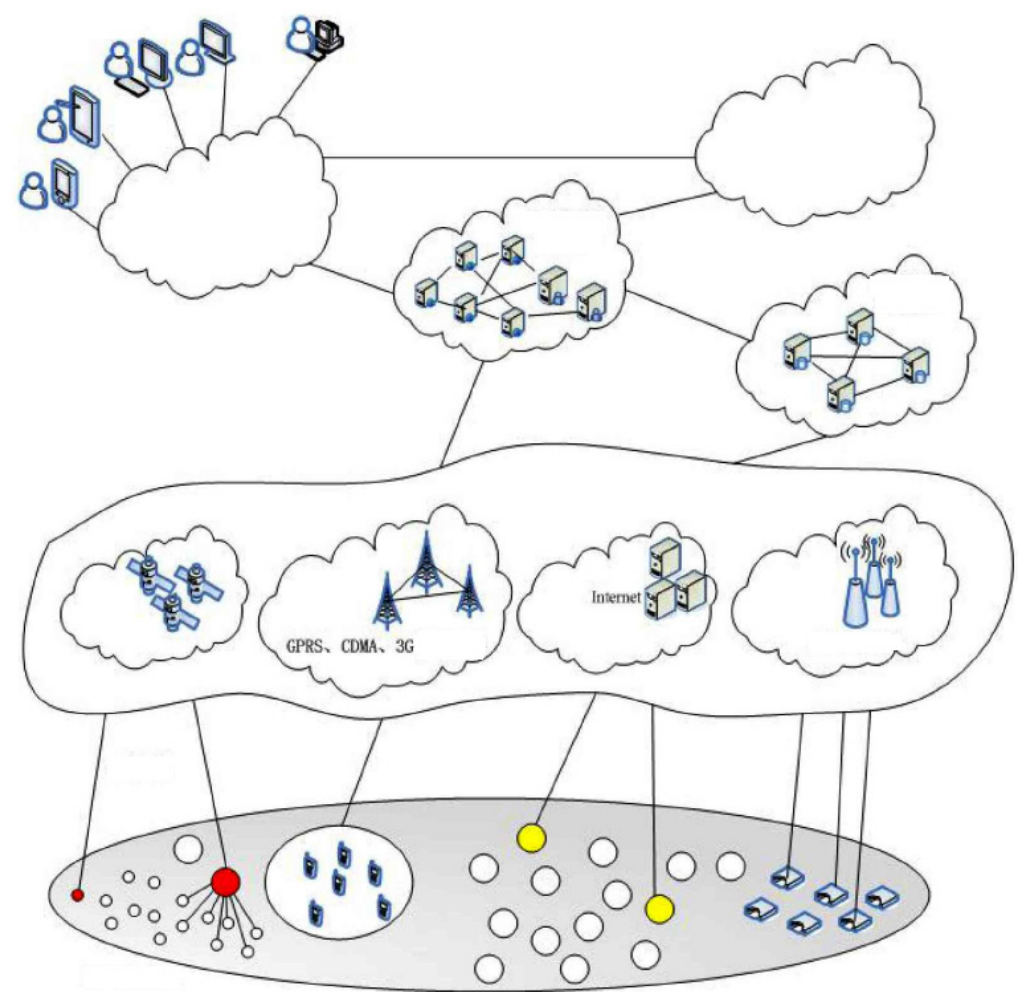

Fig. (2). IOT physical deployment.

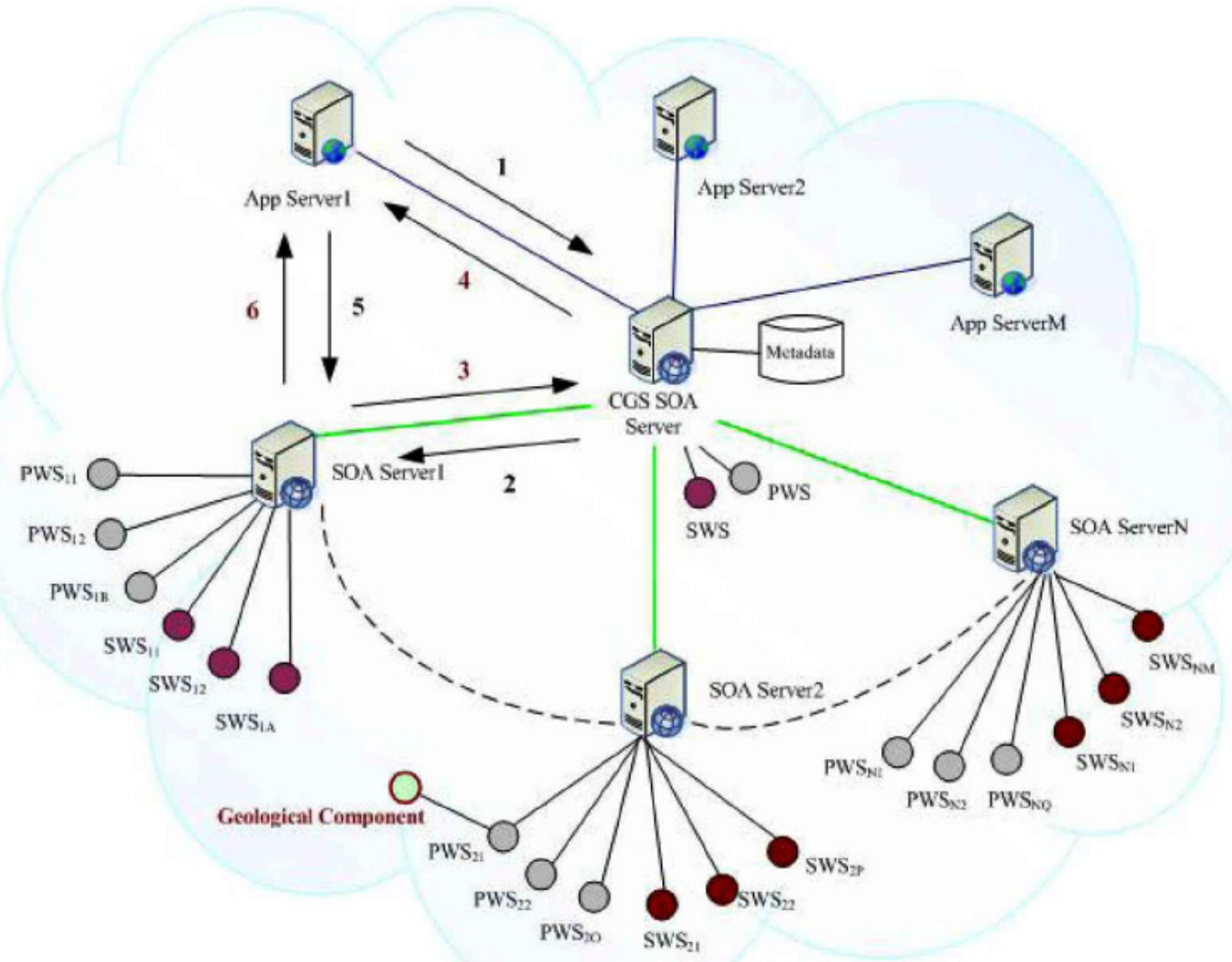

Fig. (3). Cloud computing service center. 


\section{LOGISTICS INFORMATION SYSTEN BASED ON IOT AND CLOUD COMPUTING}

\subsection{The Importance of Logistics Information System}

The 7 magnitude earthquake occurred in Lushan County of Ya'an City Sichuan eight two on April 20, 2013, 3 hours later the rescue of 7 times Yushu earthquake was mobilized to the forth strength. The national relief system substantially rebuilt after the Wenchuan earthquake, was showing the timely response and strong ability to mobilize; examined by Yaan earthquake The country's emergency response capacity had been greatly improved. Since disaster localities was much in the mountains often leading communications power outages road houses destroyed the disaster situation was difficult to grasp at the first time so it was difficult to carry out emergency relief work. When disasters happened, the emergency logistics information system based on Internet of things could play a significant role in the whole disaster relief for instance, urgent and co-ordination dispatching food, water, medicines, tents and other supplies; summary reporting disaster situations; optimal scheduling emergency vehicles; optimal planning the way forward and so on. When these emergency logistics work was quickly and efficiently carried out in a disaster it provided good protection for a series of disaster reduction, rescue victims, reconstruction and so on.

\subsection{Analysis Process}

In this context, the paper mainly discusses how to construct the emergency logistics information system based on Internet of things and researches the evaluation of the overall information system. The planning, analysis, construction, implementation and evaluation of emergency logistics information system based on Internet of things are discussed one by one. [5]

First of all, this paper summarizes the research achievements of predecessors, generalizes and reviews of the previous research papers. Based on the current research status the overall planning for the emergency logistics information system based on Internet of things is given; the construction goal of information system is put forward, and the system's technical economic, social feasibility and necessity is analyzed. At the same time the preliminary investigation and analysis are done to the running situation of the current information system.

The information system is analyzed in detail in paper starting from the needs and functions of information system, the information system of business process is analyzed too. Combining with the system requirements and business process the system's overall view and four layers architecture diagram are given; the system running environment and the corresponding development models and tools are studied. Later, each subsystem and the function module of the system are divided and elaborated and the code is designed. On the basis, the concept, the logical and physical model of database are designed, and the database subsystem is carried out, achieving the goal of the overall building of information system.
Finally, the paper discusses the implementation and security factors of the information system, four suggestions are put forward. By choosing scientific and reasonable evaluation method and index, the author makes an evaluation of the information system; and three actual emergency logistics information system are selected to evaluate, then the results of the evaluation are given. In addition, six constructive views are put forward to the emergency disaster relief itself, expecting to offer reference to the improvement of our Country's emergency logistics.

\section{KEY TECHNOLOGIES}

Nowadays, Big data has become an important direction of development of modern information technology, and sharing and analysis of big data would not only bring immeasurable economic value, but also play a significant role in promoting the development of society. Big Data-as-a-Service (BDaaS) is a new data resource usage pattern and a new form of service economy, by encapsulating heterogeneous data; it can provide ubiquitous service consumers, standardization, on-demand services, including search, analysis or visualization.

Due to the research of BDaaS is in the conceptual discussion stage, it still faces four challenges: 1)There is no standardized, user experience based BDaaS architecture which can shield the complexity of data sources and operations; 2)The lack of generic unstructured data model which reflects user behavior characteristic, made BDaaS for unstructured data diff cult to build; 3)Existing data model follows the Web services model, however, so far, holistic BDaaS service model with the characteristics of big data has not yet appeared; 4)There is no appropriate solution in providing data retrieval, analysis and visualization services and optimizing service capacity. The algorithm is shown in Fig. (4), Eq. (1) and (2).

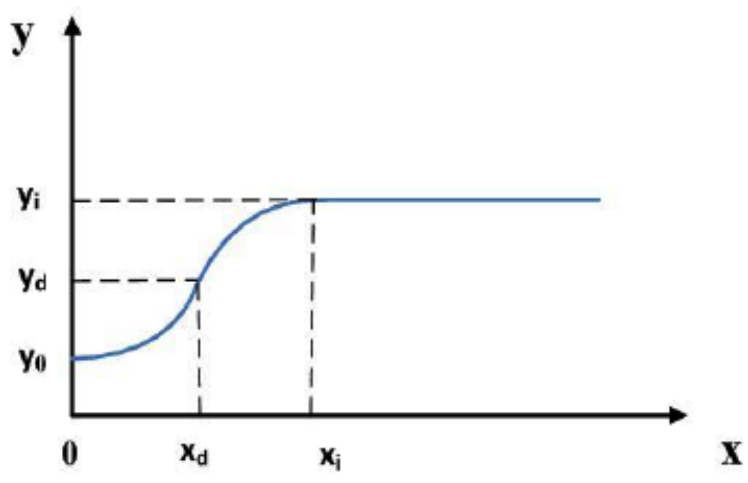

Fig. (4). Algorithm figure.

In order to solve the above problems, four key technologies of BDaaS architecture, data model, BDaaS service model, as well as BDaaS applications will be in-depth study. Firstly, this paper designed a User Experience-oriented BDaaS Architecture, so as to provide a high level of standardization guidance for building a platform. Secondly, in terms of the data model, in order to unify description unstructured data, the user behavior-based unstructured data model has been designed. Thirdly, in terms of the service model, algebraic model has been established by using proc- 


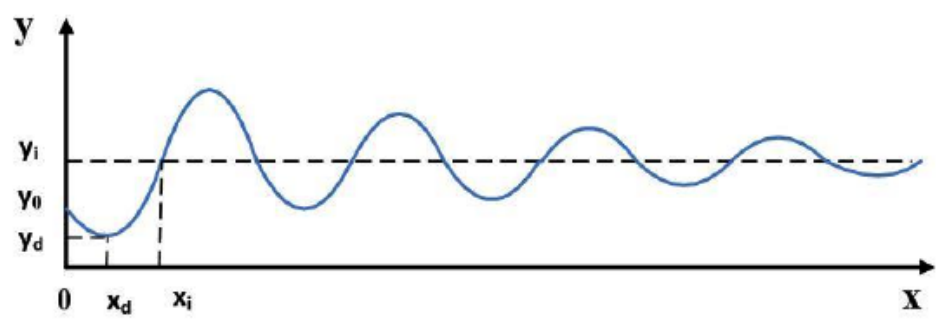

Fig. (5). Algorithm result.

ess algebra, and then extended OWL-S ontology-based BDaaS model and the service composition approach were designed. Finally, service processes of retrieval, analysis and visualization have been described in detail, and the two measures of improving the retrieval services accuracy and service efficiency have been used to optimize the BDaaS capacity.

(1) As existing unstructured data models were difficult to meet the demand for BDaaS, the Galaxy Data Model (GDM) has been proposed, which is a user behavior based unstructured data model. By monitoring the behavior of data generator people, a generic model with fully attributes like user behavior, semantic background have been created, which is the basis for the realization of the BDaaS for unstructured data. The case study shows GDM not only has good versatility and comprehensiveness, but also has a lightweight, easyto-use description language and operating language. In addition to the traditional file system, GDM also supports modeling and retrieval of unstructured data in HDFS. In addition, GDM has application in the National Pre-pregnancy Check Information Management System (NPCIMS) to verify its feasibility and practicality.

$$
y=\left\{\begin{array}{cc}
y_{0} & , x=0 \\
\arctan \left(x-x_{d}\right)+y_{d}, & 0<x<x_{i} \\
y_{i} & , x_{i}<x
\end{array}\right.
$$

(2) Due to the holistic BDaaS service model with the characteristics of big data has not yet appeared, Extended OWL-S based Big Data-as-a-Service model (EO-BDaaS) has been proposed. By add properties of the data sources, data types, service operation in the OWL-S in order to build many types of BDaaS such as search, analysis, visualization, and to compose service dynamically. Case study shows, compared with the existing data services, EO-BDaaS with a more comprehensive description on attributes and operations. Besides, it has capabilities such as strong semantic comprehension and automatic service composition, and integrated the unique combination operations of BDaaS into the implementation of data services seamlessly as shown in Fig. (5).

(3) To solve the problem of low accuracy of retrieval services, this paper presents the heat sensitive unstructured data retrieval ranking algorithm HotRank. First heat score was calculated, which is the match degree between the tasks attributes of search results and task attributes of services consumers, after that assigned the scores to each of the search results, and then sorted search results based on heat score. By using such means to make search results more in line with the preference of the user. The simulation results show that, the Precision-Recall of HotRank is better than Windows Search ranking algorithm. Therefore as the improving of retrieve accuracy, HotRank is able to optimize not only the user experience, but also the service capacity.

$$
y=\frac{\sin \left(x-x_{i}\right)}{x}+y_{i} \quad, x>0
$$

(4) A data heat recognition-based Hybrid Prefetch Algorithm (HPA) has been proposed to meets the quickly respond requirements of the BDaaS. First, by analyzing the log of user data operation and develop data heat determine rules, then according the dynamic and static prefect rules to get candidate data, finally prefect data would be take into the cache. The simulation results show that average hit rate of HPA is $55 \%$, the average accuracy rate of HPA is $43 \%$, which indicates that the algorithm not only has good ability to predict user operation of data, but also to optimize the BDaaS capacity. In addition, HPA-based Hybrid Prefect based Persistent Caching architecture has been applied in the National Pre-pregnancy Check Management Information System (NPCMIS) in order to verify its effectiveness. The specific calculation process is shown in Eq. (3), (4) and (5).

$$
\frac{\partial S}{\partial t}=\left\{\begin{array}{cc}
\beta \omega(t)+S_{01} & , 0<\omega<\omega_{0} \\
\ln [\delta(t)+1]+S_{02} & , 0<\delta<\delta_{0} \\
\arctan \left[\varepsilon(t)-\varepsilon_{d}\right]+S_{d} & , \quad o<\varepsilon<\varepsilon_{0} \\
\frac{\sin \left[\gamma(t)-\gamma_{i}\right]}{\gamma(t)}+S_{\mathrm{i}} & , \quad \gamma>0 \\
b \eta^{2}(t)+\sum S_{n} & , \eta>0
\end{array}\right.
$$

$$
\begin{aligned}
& \frac{d q_{1}}{d t}=\beta q_{1}+a_{1} q_{5} \\
& \frac{d q_{2}}{d t}=\ln \left(q_{2}+a_{2}\right)+a_{3} q_{5} \\
& \frac{d q_{3}}{d t}=\arctan \left(q_{3}-a_{4}\right)+a_{5} q_{5} \\
& \frac{d q_{4}}{d t}=\frac{\sin \left(q_{4}-a_{6}\right)}{q_{4}}+a_{8} q_{5} \\
& \frac{d q_{5}}{d t}=m_{1} q_{5}^{\alpha}+m_{2} q_{1}+m_{3} q_{2}+m_{4} q_{3}+m_{5} q_{4}
\end{aligned}
$$


Internet of things (IOT), an application of the next generation Internet, has received much attention and will be a worldwide competition emphasis because IOT can realize the interconnection between things and things, also between things and people. IOT will improve old industries and promote new ones. The popularization and application of IOT will not only influence the progress of industrialization and informationization, but also have far reaching impact on social economy and human life. The construction of modern logistics service system should be sped up to correspond with the development of informationization. Modern logistics should be the new force to promote the development of modern services as it has quicker reaction, integrated function, serialization service, normalized work, systematic target, modern method, networked organization, marketed organization and electrified information. It is of great theoretical value and realistic meaning to study the multi dimensional synergy of logistic management in IOT environment.

$$
\left\{\begin{array}{l}
q_{5}=0.0678 \\
q_{5}=-0.1720 \\
q_{5}=-0.1037 \\
q_{5}=0.0506
\end{array}\right.
$$



Fig. (6). Result.

The result figure is show in Fig. (6). Regarding to the need of multi-dimensional synergy in modern logistics in IOT system, the modern logistics order parameter equation is built and solved. Multi-dimensional synergetic study of modern logistics is proposed on the basis of sensing layer, network layer, application layer and management layer of IOT and from the four-dimensional synergetic demand by technology synergy, information synergy, service synergy and management synergy. This paper consists of theory basis, technical basis, application examples and application management. The studied in this paper is of scientific significance and practical significance for the need of multidimensional synergy in modern logistics in IOT environment.

The Internet of things (IOT) is considered to be the next step in the revolution of internet. People are expecting to be able to quickly connect between objects existed all the time no matter where. This requirement not only propels rapid development of IOT technology and wide acceptance of RFID devices, but also deviates the traditional computing style to smart IOT computing. Compared to hardware and network support in pervasive computing, the reliability of sensors software for IOT, which in return, is limited the application and deployment of IOT to some extents. Unlike the traditional distributed computing software running in stable environments with rich resources, sensor nodes in IOT runs in unforeseen wireless network environments, limited resources and continuously altered context. These put a great deal of demands on the dynamic software, resource configuration and energy maintenance technology. From perspectives of service, including resource service, data transmission service and component service, this thesis mainly concentrates on the dynamic maintenance and resource configuration issues at runtime in IOT infrastructure environments. In order to minimize the resource requirements and point-topoint and end-to-end delay in a dynamic IOT environment, multiple technologies, such agent, ECA rules mechanism, game theory, graph theory, LaGrange optimization and fuzzy control are further explored and used to study problems in device service networks dynamic maintenance, dynamic software updating maintenance, dynamic resource adjusting and configuration optimization and rechargeable RFID sensor networks dynamic energy maintenance. There are Matlab result in Fig. (7)

As the inevitable trend of modern logistics, intelligent logistics updates several concepts of modern logistics from two aspects, technology and management. It also makes modern logistics have characteristics, such as informatization, network, integration, intelligent, flexible, agility, visualization and automation. And it promotes comprehensive development of the logistics industry. Developing intelligent logistics is an important means of promoting the development of modern logistics and circulation.

\section{CONCLUSION}

We provide the classification of synergy; develop modern logistics synergy order parameter equations and solve equations by the Matlab. Then the order parameter which influences the synergy of system was found out. The problem of which is the synergy key factor of logistics system was solved in this chapter.

The mobile logistics service node perception was studied based on dynamic sensing technology synergy of IOT. The mobile logistics service node discovery algorithm was designed based on algorithm was verified with Matlab clustering algorithm.

With information synergy of IOT network layer as the prerequisite, we develop the information synergy distribution simulation mode. The simulation mode can be used to comparing the distribution path and time under the station of information synergy and information no-synergy. The simulation result demonstrated the information synergy have positive effect on logistic system.

This paper consists of theory basis, technical basis, application examples and application management. The studied in this paper is of scientific significance and practical significance for the need of multi-dimensional synergy in modern logistics in IOT environment. 

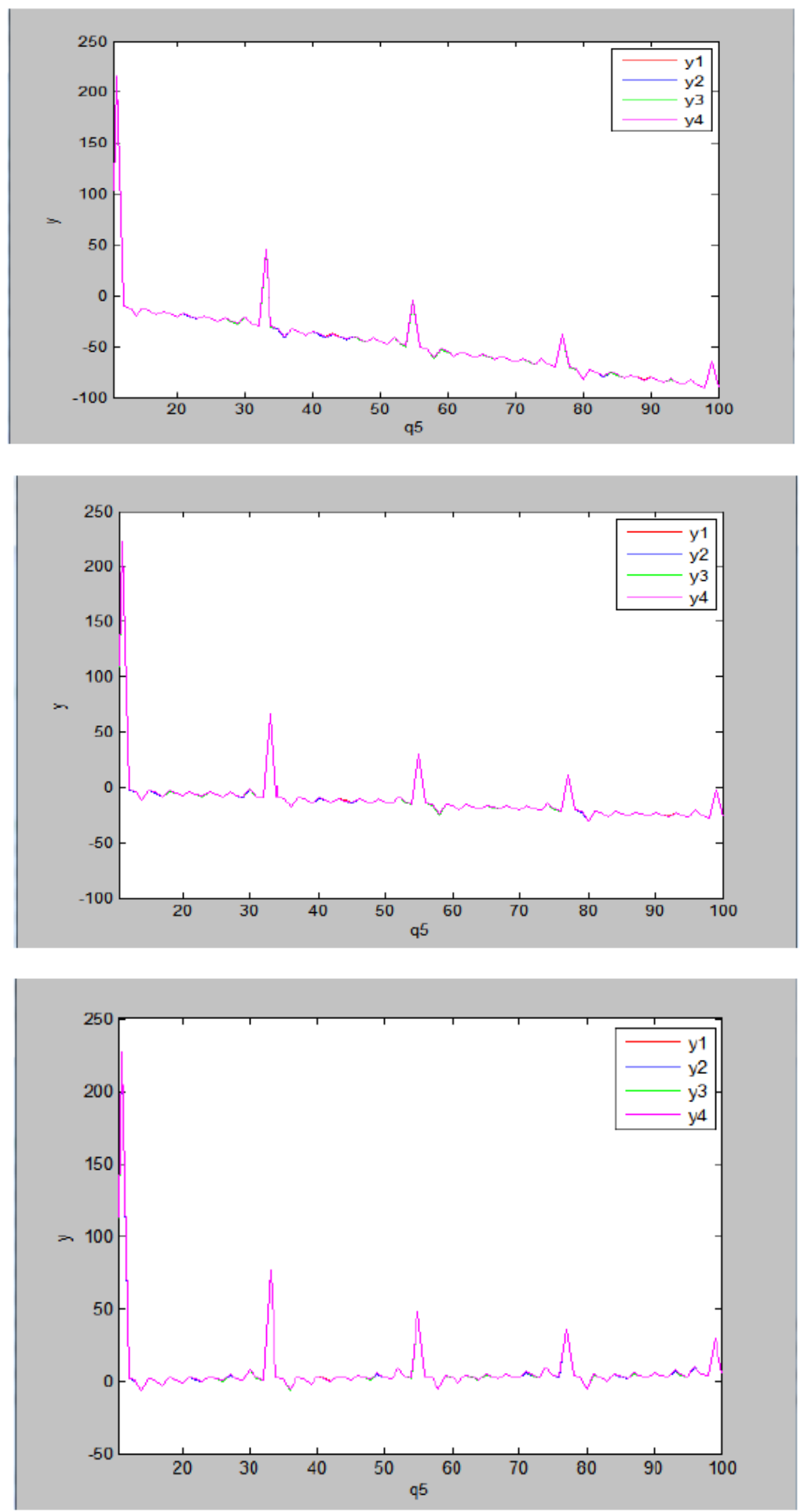

Fig. (7). Matlab result.

\section{CONFLICT OF INTEREST}

The authors confirm that this article content has no conflict of interest.

\section{ACKNOWLEDGEMENTS}

This work is supported by science project in hebei province, the nane of the project: Based on internet of things vegetables intelligent logistics management information system research and development, The number of the project: 13220319D.

\section{REFERENCES}

[1] R. Weiss, "Obama Administration Unveils 'Big Data' Initiative: Announces \$200 Milfion in New R\&D Investments," Office of Science and Technology Policy Executive Office of the President, 2012. 
[2] E. w. T. "Ngai Logistics. information systems:The Hong Kong experience". InternationalJournal of Production Economics, vol. 113, 2008, pp.223-234.

[3] R. V. Kranengurg, "The Internet of Things-A critique of ambient technology and the all-seeing network RFID".Institute of Network Cultures, 2008.
[4] T. S. Jayram, R. Krishnamurthy, S. Raghavan, et al. "Avatar information extraction system," IEEE Data Engineering Bulletin, vol. 29, no. 1, pp. 40-48, 2006.

[5] Yue W, Song M, Han J, et al. "Location Context Aware Collective Filterin Algorithm," Pervasive Computing and the Networked World. Springer Berlin Heidelberg, pp.788-800, 2013.

Received: September 16,2014

Revised: December 23, 2014

Accepted: December 31, 2014

(C) Guo and Qu; Licensee Bentham Open.

This is an open access article licensed under the terms of the (https://creativecommons.org/licenses/by/4.0/legalcode ), which permits unrestricted, noncommercial use, distribution and reproduction in any medium, provided the work is properly cited. 